\title{
Rationality and necessity of vascular stapler application during liver resection (Review)
}

\author{
ER-LEI ZHANG, ZHI-YONG HUANG and XIAO-PING CHEN \\ Hepatic Surgery Center, Tongji Hospital, Tongji Medical College, Huazhong University \\ of Science and Technology, Wuhan, Hubei 430030, P.R. China
}

Received September 1, 2020; Accepted February 17, 2021

DOI: $10.3892 /$ etm.2021.9929

\begin{abstract}
Liver resection (LR) is the primary treatment method for patients with hepatocellular carcinoma (HCC). Improving surgical safety and reducing surgical morbidity and mortality is important for patients receiving LR. Various devices have been developed to facilitate vascular transection to reduce intraoperative blood loss, which is considered to be a predictor of poor surgical outcomes in patients undergoing LR. Vascular staplers have been widely applied for the division of major vascular and biliary structures in the process of LR; however, when and how to use these tools remains controversial. This review aims to report the rationality and necessity of using vascular staplers in vessel transection during liver surgery. Due to the risk of intraoperative and postoperative hemorrhage and biliary fistula, the process of transection of the portal pedicle and hepatic vein is a crucial step during LR. Stapling represents a vascular dissection technique that is widely used in laparoscopic LR and has then been popularized in open LR. Advocates argue that stapler transection methods provide several advantages, including diminished blood loss, fewer transfusion requirements and shorter operative times. However, other studies have failed to demonstrate those benefits when using these tools compared with the simple clamp-crushing technique. Using the stapler vascular transection method resulted in smaller surgical margins and similar surgical outcomes compared with those of the clamp-crushing vascular transection method. However, the intraoperative use of vascular staplers may significantly increase the financial burden of liver resection for patients with HCC, while not improving short- and long-term outcomes. Therefore, it has
\end{abstract}

Correspondence to: Professor Xiao-Ping Chen, Hepatic Surgery Center, Tongji Hospital, Tongji Medical College, Huazhong University of Science and Technology, 1095 Jie Fang Da Dao, Wuhan, Hubei 430030, P.R. China

E-mail: chenxpchenxp@163.com

Abbreviations: LR, liver resection; HCC, hepatocellular carcinoma

Key words: vascular stapler, vessel transection, liver resection, rationality, necessity been suggested that vascular staplers should not be routinely used in LR. The current review discussed the above points and recommended that the stapling transection of the portal pedicle and hepatic vein should be applied during laparoscopic LR in a rational manner. However, the suturing ligation method should be routinely used in open LR.

\section{Contents}

1. Introduction

2. Open LR

3. Laparoscopic LR

4. Conclusions

\section{Introduction}

Liver resection (LR) is considered to be the first choice for the treatment of hepatocellular carcinoma (HCC). In the past decades, liver surgical techniques have progressed considerably with the increased knowledge of liver anatomy. The development of professional hepatobiliary centers with experienced and skilled surgeons, along with the progression of perioperative care, has allowed surgeons to safely perform LR in normal and cirrhotic livers. However, the rate of perioperative complications remains high, with morbidity and mortality rates of 14-33 and 3-5\%, respectively (1-3). Several studies have revealed that intraoperative blood loss and transfusion, which are significant for the postoperative course of patients receiving LR, are important predictors of adverse outcomes $(4,5)$.

It may be speculated that the technical advancements in methods aiming to reduce blood loss may further improve the surgical outcomes of patients. Among these methods, a vascular stapler is commonly used for vascular division due to its safety, simple use, shortening of the surgical duration and similar or improved outcomes compared with traditional suture techniques $(2,5-7)$. In recent years, vascular staplers have been widely applied to the pulmonary artery, pulmonary veins and bronchus transection during thoracic surgery (8). In liver surgery, vascular staplers have also been used for the transection of hepatic veins or Glisson's pedicles. The usefulness of this device has been previously reported (9). Vascular staplers are able to control hepatic veins and Glisson's pedicles prior to parenchymal transection, thus reducing major liver resection-associated hemorrhage, decreasing blood loss and 
minimizing the requirement for blood transfusions. In addition, this decreases the need for portal triad clamping during liver ischemic reperfusion injury $(2,10)$. Previous systemic reviews proposed that stapler devices should be used in open or laparoscopic LR and reported that up to nine vascular staplers are used for parenchymal transection in laparoscopic liver surgery $(7,11)$. Using vascular staplers may improve the speed and safety of ligation in both inflow and outflow vessels, thereby facilitating LR $(12,13)$. However, the use of vascular staplers adds to the financial burden of patients undergoing LR. Accordingly, decreasing medical costs while reducing perioperative morbidity and mortality should be pursued by liver surgeons. Whether hepatic pedicle branches and hepatic veins should be transected with a vascular stapler or via suturing ligation during major liver resection remains controversial. Furthermore, there is no standard method for the closure of portal pedicles or hepatic veins during open and laparoscopic LR.

The present review aimed to outline and assess the current application of vascular staplers for hepatic vessel transection in open and laparoscopic LR from a financial perspective and to provide reasonable advice for vascular application in clinical settings.

\section{Open LR}

As the liver is a vasculature-rich organ, the safe and effective dissection of liver vessels and parenchyma without causing biliary injury and ensuring minimal blood loss are the main aims when performing LR. Several factors may affect the extent of blood loss and perioperative outcomes of patients, including the extent of LR, whether tumors are located near portal pedicles, the inferior vena cava or hepatic veins, tumor size and the technique applied to transect the main hepatic vessels $(14,15)$. Among these factors, hepatic vessel transection is the only one that may be controlled. Suturing ligations or stapling the hepatic pedicles and veins during hepatic parenchyma transection remains controversial. Pringle's maneuver is primarily used to occlude vascular inflow to the liver when performing suturing ligation during transection. This is a relatively low-cost procedure. However, liver surgeons are prone to using vascular staplers to limit transection durations and shorten the portal pedicle closure time, or they may even perform no occlusion $(7,16)$. Transection of the hepatic veins or Glisson's pedicles using vascular staplers has been widely used in multiple surgical liver centers $(2,9,10,17)$. As early as 1989 , vascular staplers were used in open LR to control hepatic veins within the hepatic parenchyma (18). When the precise isolation of hepatic veins is difficult to determine due to their depth within the tissue, vascular staples may be placed for transection. Furthermore, vascular staplers have also been used to dissect hepatic pedicle branches. They are able to control blood inflow to the partial liver prior to hepatic parenchymal transection and efficiently reduce major hepatic resection-associated hemorrhage, thereby reducing the requirement to perform Pringle's maneuver $(19,20)$.

The portal vein, the hepatic artery, the bile duct and connective tissues surrounding them form Glisson's system, which extends up to the liver parenchyma. Couinaud introduced three main methods for the inflow system at the first hepatic hilus: The intrafascial, the extrafascial, and the extrafascial combined with the trans-fissural method (13). The conventional dissection for separating the elements in the hepatoduodenal ligament is regarded as the intrafascial approach. The intrafascial approach, which was introduced for right hepatectomy in the early 1950s, separately ligates the hemi-Glisson's pedicle (21). For a certain time, the intrafascial approach was considered to be a standard procedure during anatomical hemi-hepatectomy. However, Bismuth and Couinaud determined that the intrafascial approach carried a high risk of vessel and bile-duct injury in the remaining liver, resulting from the incorrect identification of anatomical variations, which was a major concern during LR $(13,22)$. If the main portal fissure or left supra-hepatic fissure is opened after liver parenchyma dissection, the surgeon may confirm the presence of Glisson's pedicles, which arise from the hilar plate or the umbilical plate. This procedure is called the extrafascial and trans-fissural approach and was introduced in the 1960s $(23,24)$. When the hilar plate is pulled down after detaching the liver parenchyma, the right and left Glisson's pedicles may be easily identified $(12,25)$. Using this approach, vascular staplers may easily transect Glisson's pedicles without dissecting the portal vein, hepatic artery and bile duct. The key element of the extrafascial approach is to confirm the existence of Laennec's liver capsule. The fiber configuration of Glisson's capsule and the liver capsule differ. Therefore, Glisson's pedicles may be separated from Laennec's capsule, allowing for the main, sectional and segmental hepatic pedicles to be approached at the first hepatic hilus without liver dissection (26). The extrafascial Glisson's approach is a simple and popular procedure for anatomical LR. However, this method may result in occasional injury to the small branches of the hepatic pedicles and it does not succeed if the tumor invades or is close to the hepatic pedicle (21).

The application of vascular staplers may simplify the transection of Glisson's pedicle, decrease blood loss and reduce the duration of surgery. Previous studies have revealed that operative times were $>20$ min longer when using the hilar dissection method compared with the Glisson's pedicle approach using vascular staplers. Without the application of vascular staplers, the hilar dissection method must dissect all the elements of the pedicle and close the element stumps using a running suture or double ligature to avoid slippage of the ligated string $(17,27)$. In addition, the Glisson's approach may be more suitable to transect the pedicle in patients with scars, which are difficult to dissect, making en bloc dissection much safer and easier (28). Anatomic variations should be considered during LR. Any variations in the arteries and bile ducts of the liver frequently occur under the hilar plate. Therefore, transection under the hilar plate is difficult and dangerous (12). The confluence of the left and right bile ducts may be accidentally ligated while performing stapling of the main right or left pedicle. Lowering the hilar plate in combination with counter traction is therefore necessary to prevent this complication. Furthermore, in $60 \%$ of cases, the left and middle hepatic veins join to form a common stem into the vena cava (22). Thus, the middle hepatic vein should be protected when stapling the left vein. In addition, if not all of the branches of the caudate lobe are protected during left hepatectomy, bile ducts from the caudate lobe, which frequently drain into the left bile duct, may be injured by the vascular stapler (29). 
Assessment of techniques that use stapling devices during LR suggested that they are useful in the safe transection of inflow and outflow hepatic vessels (30). When tumors occupy hepatic pedicle branches or hepatic veins, vascular staplers are recommended to divide both the hepatic pedicle and hepatic veins to control the hepatic inflow and outflow prior to hepatic parenchyma transection. This technique also ensures radical resection of tumors with minimal blood loss. Taking this into account, surgeons have not been required to routinely apply Pringle's maneuver or other forms of vascular control during LR. However, there are certain disadvantages to the application of vascular staplers. First, serious blood loss may occur when the stapler has sealed only half the diameter of the vessels. Furthermore, traction injury or tear of the inferior vena cava may occur while placing the stapler during the transection of hepatic veins. A previous study has reported that the silicone tube-guided vascular stapler technique is a simple and feasible approach that avoids vascular injury during the division of major vascular structures when performing LR (10). Accidental ligation of the reserved vessel structures may also occur when using vascular staplers. To avoid the accidental ligation of the biliary confluence when stapling the right or left pedicle, firm countertraction should be applied to the tape (19). Furthermore, during stapler-assisted hepatectomy, failure to release or misfire the staplers is another adverse event that may lead to serious and life-threatening hemorrhage. If such an incident occurs, a vascular clamp should be used to control bleeding. Such cases have been reported sporadically during LR, indicating their low occurrence rate (30). However, care should be taken to ensure that no vascular clips are placed near the site of staples.

The development of surgical instruments has raised questions regarding whether the high cost of vascular stapling devices is justified if better surgical outcomes are demonstrated in patients undergoing LR using the simple clamp-suturing technique. From an economic standpoint, application of the clamp-suturing technique is associated with low cost and easy disposal of material. The cost-effective advantage of this technique therefore appears obvious. Furthermore, in situations of limited health care resources, surgeons are under pressure to provide the best possible treatment with minimal expenditure. When comparing average intra-operative costs in developed countries (which include surgery, anesthesia, blood products and the average number of vascular staplers), it was determined that there was a cost-benefit when using stapler hepatectomy instead of conventional methods (31). A vascular stapler costs only 101.64 USD and each reload comes to 51.33 USD in Western countries (2). However, in China, the price of a vascular stapler and reload is $>8$ times higher than that in Western countries. Introducing such expensive stapling devices therefore appears unjustified in China.

The stapler-ligation technique may therefore not be recommended if visualization of the main hepatic vein is obscured due to the larger size or special location of a liver tumor, as suture repair at the caval side of hepatic veins cannot be performed easily if the vascular staplers fail to fire. As mentioned above, hilar dissection should be performed if the tumor is situated close to the portal bifurcation, as this technique may increase the oncological safety margin (29). To conclude, suture ligation of the hepatic pedicles or veins is a straightforward technical approach for open LR (32). The use of vascular staplers rarely adds to the ease or speed of transection, but certainly adds to the overall medical cost in China.

\section{Laparoscopic LR}

Laparoscopic LR is a common procedure that has been frequently used worldwide (33-36). As a less invasive method compared to open LR, several studies have indicated that laparoscopic LR demonstrates marked superiority with regard to intraoperative blood loss, postoperative recovery and hospital stay in certain selected patients, such as those with tumors located at the edge of the liver (37-40). Laparoscopic LR has been gradually accepted for use in local tumor resection, wedge resection, left lateral segmentectomies and major hepatic resections (40-43). Increasingly complex surgeries, such as extended right or left hepatectomy, mesohepatectomy and two-stage LR, have been gradually performed by experienced surgeons in hepatic surgery centers $(11,44,45)$. Rubber tape is routinely placed around the hepato-duodenal ligament to allow for Pringle's maneuver to be performed if necessary. The elements of the portal triad may require to be individually dissected for selective ligation and suture during major LR. In certain complex cases, laparoscopic LR requires elaborate vessel and biliary tract reconstruction, which is usually difficult to perform in laparoscopic settings (46). Under such circumstances, vascular stapling devices may safely and rapidly divide vascular and biliary structures without suturing and ligating, which makes this complex procedure comparatively easier and safer. Vascular staplers have been routinely used during laparoscopic LR and have been proven to be a convenient and effective method of vascular control $(44,47)$. In the laparoscopic stapling technique, the hepatic parenchyma is thinned out by harmonic devices, after which the vascular staplers are used to transect the remaining hepatic parenchyma, larger hepatic vessels and Glisson's pedicles. Scuderi and Troisi (44) compared 35 laparoscopic LRs using vascular staplers with 57 laparoscopic LRs without vascular staplers. Although the tumor size was significantly larger in the stapler group compared with the traditional group, the results demonstrated that the mean surgical times and degree of blood loss were similar between the two groups. Pringle's maneuver was also used less frequently and the blocking time of the portal triad was shorter in the stapler group. Finally, the conversion and morbidity rates were relatively lower, suggesting that the use of vascular staplers for dividing intrahepatic vessels and biliary structures was safe and feasible in laparoscopic LR (44).

In recent years, an increasing number of studies have advocated the use of vascular staplers in hepatic parenchymal transection, which have been recommended as safer alternatives to parenchymal dissection in laparoscopic LR $(11,44)$. However, an additional study demonstrated that hepatic parenchymal transection using vascular staplers may result in more complications, including hemorrhage and bile leakage (48). Although certain liver surgeons have advocated transection of the hepatic parenchyma with laparoscopic vascular staplers, laparoscopic bipolar or monopolar electrocoagulation in addition to either harmonic scalpel or bi-clamp is routinely used for patients receiving LR. Furthermore, for tumor enucleation, wedge resection or segmentectomy of the liver in laparoscopic 
LR, stapling parenchymal transection may result in unnecessary transection of the surrounding liver parenchyma through the proposed division line.

The laparoscopic stapling device is particularly useful for left hepatectomies and left lateral lobectomies, as the left pedicle is the thickest and therefore requires careful transection of the portal triad $(42,46,47)$. Furthermore, the left branch of the portal vein is extended before it trifurcates and the structures of the portal triad-supplying segments two and three may be easily located in the umbilical fossa. The laparoscopic stapling device is inapplicable for transection of the right portal triad, as the structures frequently bifurcate early into anterior and posterior branches $(11,22)$. Furthermore, due to the angle of these structures, it is often necessary to place the distal ends of the stapler into the underlying hepatic parenchyma. This causes unnecessary blood loss and increases the risk of injuring deeper structures (45). For an extended right hepatectomy, the branches of segment four deriving from the left hepatic triad must be located, meaning that this cannot be performed directly by the vascular stapler and should be dissected separately $(11,48)$. This is also the same when this structure needs to be taken for mesohepatectomy (44).

The extrafascial approach is more widely used in laparoscopic LR. Laennec's capsule may facilitate this approach in laparoscopic hepatectomy due to its magnified view. When performing laparoscopic major hepatectomy, the whole portal triad is usually required to be located and transected using laparoscopic stapler devices (24). The en bloc transection of hepatic pedicles using vascular staplers may be faster than the isolated ligature of each element of the pedicle, particularly in laparoscopic settings; the stumps of Glisson's pedicle may be continuously sutured via 4-0 prolene if required (49). Furthermore, the procedure may avoid bleeding from an injury of collateral vessels in Glisson's pedicle. However, a considerable complication that occurs when using the vascular stapler is the accidental stapling of the biliary confluence, which may cause postoperative biliary stenosis or bile leakage, presenting as obstructive jaundice or bile fistula (19). To avoid accidental injury of the bile duct, a firm counter traction by taping or stapling at the second branch is applied, which avoids further severe complications. Another method to prevent postoperative biliary stenosis or bile leakage is the location and isolation of the biliary duct for major right and extended hepatectomy (50). Occasionally, it may be difficult to locate hepatic bile ducts. As a result, when a suspicious bile duct is due for isolation, it is transected with a laparoscopic shear until bile flows out for verification, after which the bile duct is over-sewed with absorbable sutures. As mentioned above, due to the length of the left hepatic pedicle, there is no requirement to routinely isolate the biliary tracts when using vascular staplers. It is hypothesized that postoperative bile leaks in major right hepatectomy may be reduced by identifying the biliary system individually (27). In circumstances where the tumor lies close to the right or left hepatic pedicle during hepatectomy, vascular staplers should not be used, as tumor margin violation may occur near the portal pedicle (16). In laparoscopic subsegmentectomy, a substitute method is the identification and division of intrahepatic Glisson's pedicles. Careful dissection and proper surgical control of all vascular structures are crucial for performing safe and successful laparoscopic LR.
A disadvantage of applying vascular staplers in laparoscopic LR is the increased additional cost of disposable devices (51). This is particularly important as laparoscopic surgery is more expensive than open surgery. It has been reported that up to three vascular staplers are required when used for vascular pedicle control (2). Other studies have indicated that the additional costs of vascular staplers may be offset by shorter operating times and lengths of hospital stay in patients receiving laparoscopic LR (48). As vascular staplers are particularly expensive in China, liver surgeons should assess the benefit of convenient hepatic vessel transection against the additional costs that arise from using multiple reloads for hepatic pedicle or hepatic vein transection. Besides the economic aspects of using multiple vascular staplers, the disadvantages of applying these tools in laparoscopic LR are more likely to occur when compared with open LR. Therefore, the necessity of vascular stapler application in laparoscopic LR should be reasonably evaluated. From a surgical perspective, the only contraindication to a laparoscopic LR is the surgeon's inability to perform the procedure. Unskilled and inexperienced liver surgeons may resort to the use of surgical instruments in laparoscopic LR and thus add the additional costs $(40,43,52)$.

Liver cirrhosis occurs in $>80 \%$ of patients with HCC that undergo LR in China $(3,53)$. For patients with HCC exhibiting large tumors and severe liver cirrhosis, preserving adequate liver function during LR is important for patient survival, as postoperative complications frequently cause liver failure in circumstances where it is inconvenient to safely perform laparoscopic anatomic LR using vascular staplers $(54,55)$. Therefore, laparoscopic stapling during LR is limited to select cases (56).

\section{Conclusions}

Vascular staplers have been widely applied to major vascular and biliary structures in open and laparoscopic LR. In China, vascular stapling devices are more expensive than in Western or developed countries. Due to limited health care resources, liver surgeons are under great pressure to provide the best possible treatment with minimal expenditure. The present study recommends that stapling transection of the portal pedicle and hepatic veins should be reasonably applied in laparoscopic LR, whereas the suturing ligation method should be routinely used in open LR. Almost all published studies on this topic are retrospective, providing an objective analysis of the current application of vascular staplers in open and laparoscopic LR. However, more randomized controlled studies based on cost-effective analysis are required to clarify the benefits and indications of the future application of stapling devices for the transection of hepatic vessels.

\section{Acknowledgements}

The authors thank Dr Wajeehullahi Akilu from Tongji Medical College for language editing and revising this manuscript.

\section{Funding}

No funding was received. 


\section{Availability of data and materials}

Not applicable.

\section{Authors' contributions}

ELZ and ZYH wrote the manuscript and XPC provided senior critical revision. ELZ and XPC confirmed the authenticity of all the data. All authors read and approved the final manuscript.

\section{Ethics approval and consent to participate}

Not applicable.

\section{Patient consent for publication}

Not applicable.

\section{Competing interests}

The authors declare that they have no competing interests.

\section{References}

1. Huntington JT, Royall NA and Schmidt CR: Minimizing blood loss during hepatectomy: A literature review. J Surg Oncol 109: 81-88, 2014.

2. Raoof M, Aloia TA, Vauthey JN and Curley SA: Morbidity and mortality in 1,174 patients undergoing hepatic parenchymal transection using a stapler device. Ann Surg Oncol 21: 995-1001, 2014.

3. Zhang B, Zhang B, Zhang Z, Huang Z, Chen Y, Chen M, Bie P, Peng B, Wu L, Wang Z, et al: 42,573 cases of hepatectomy in China: A multicenter retrospective investigation. Sci China Life Sci 61: 660-670, 2018.

4. Yamamoto J, Kosuge T, Takayama T, Shimada K, Yamasaki S, Ozaki H, Yamaguchi N, Mizuno S and Makuuchi M: Perioperative blood transfusion promotes recurrence of hepatocellular carcinoma after hepatectomy. Surgery 115: 303-309, 1994.

5. Kooby DA, Stockman J, Ben-Porat L, Gonen M, Jarnagin WR, Dematteo RP, Tuorto S, Wuest D, Blumgart LH and Fong Y: Influence of transfusions on perioperative and long-term outcome in patients following hepatic resection for colorectal metastases. Ann Surg 237: 860-870, 2003.

6. Dagher I, Proske JM, Carloni A, Richa H, Tranchart H and Franco D: Laparoscopic liver resection: Results for 70 patients. Surg Endosc 21: 619-624, 2007.

7. Yao DB and Wu SD: Application of stapling devices in liver surgery: Current status and future prospects. World J Gastroenterol 22: 7091-7098, 2016.

8. Szwerc MF, Landreneau RJ, Santos RS, Keenan RJ and Murray GF: Minithoracotomy combined with mechanically stapled bronchial and vascular ligation for anatomical lung resection. Ann Thorac Surg 77: 1904-1910, 2004.

9. Yanaga K, Nishizaki T, Yamamoto K, Taketomi A, Matsumata T, Takenaka K and Sugimachi K: Simplified inflow control using stapling devices for major hepatic resection. Arch Surg 131: 104-106, 1996

10. Hicks CW and Choti MA: Application of a Penrose drain guide for vascular stapling during hepatic surgery: How I do it? J Gastrointest Surg 18: 411-413, 2014.

11. O'Rourke N and Fielding G: Laparoscopic right hepatectomy: Surgical technique. J Gastrointest Surg 8: 213-216, 2004.

12. Yamamoto M, Katagiri S, Ariizumi S, Kotera Y and Takahashi Y: Glissonean pedicle transection method for liver surgery (with video). J Hepatobiliary Pancreat Sci 19: 3-8, 2012.

13. Couinaud C: Surgical Anatomy of the Liver Revisited. Selfprinted, Paris, 1989.

14. Jarnagin WR, Gonen M, Fong Y,DeMatteo RP, Ben-Porat L, Little S, Corvera $\mathrm{C}$, Weber $\mathrm{S}$ and Blumgart LH: Improvement in perioperative outcome after hepatic resection: Analysis of 1,803 consecutive cases over the past decade. Ann Surg 236: 397-407, 2002.
15. Poon RT: Current techniques of liver transection. HPB (Oxford) 9: 166-173, 2007.

16. Figueras J, Lopez-Ben S, Lladó L, Rafecas A, Torras J, Ramos E, Fabregat J and Jaurrieta E: Hilar dissection versus the 'glissonean' approach and stapling of the pedicle for major hepatectomies: A prospective, randomized trial. Ann Surg 238: 111-119, 2003.

17. Mehrabi A, Hoffmann K, Nagel AJ, Ghamarnejad O, Khajeh E, Golriz M and Büchler MW: Technical aspects of stapled hepatectomy in liver surgery: How we do it? J Gastrointest Surg 23: 1232-1239, 2019.

18. Voyles CR and Vogel SB: Hepatic resection using stapling devices to control the hepatic veins. Am J Surg 158: 459-460, 1989.

19. Fong Y and Blumgart LH: Useful stapling techniques in liver surgery. J Am Coll Surg 185: 93-100, 1997.

20. Jurim O, Colonna JO II, Colquhoun SD, Shaked A and Busuttil RW: A stapling technique for hepatic resection. J Am Coll Surg 178: 510-511, 1994

21. Honjo I and Araki C: Total resection of the right lobe of the liver; report of a successful case. J Int Coll Surg 23: 23-28, 1955.

22. Bismuth H: Surgical anatomy and anatomical surgery of the liver. World J Surg 6: 3-9, 1982.

23. Ton That $\mathrm{T}$ and Nguyen Duong Q: A new technique for operating on the liver. Lancet 281: 192-193, 1963.

24. Kasai M, Kimura S, Sasaki M and Ouchi H: Successful total right hepatic lobectomy for primary hepatoma in an infant. Surgery 54: 351-355, 1963.

25. Takasaki K: Glissonean pedicle transection method for hepatic resection: A new concept of liver segmentation. J Hepatobiliary Pancreat Surg 5: 286-291, 1998.

26. Sugioka A, Kato Y and Tanahashi Y: Systematic extrahepatic Glissonean pedicle isolation for anatomical liver resection based on Laennec's capsule: Proposal of a novel comprehensive surgical anatomy of the liver. J Hepatobiliary Pancreat Sci 24: 17-23, 2017.

27. Cresswell AB, Welsh FK, John TG and Rees M: Evaluation of intrahepatic, extra-Glissonian stapling of the right porta hepatis vs. classical extrahepatic dissection during right hepatectomy. HPB (Oxford) 11: 493-498, 2009.

28. Karamarkovic A, Doklestic K, Milic N, Djukić V, Bumbasirević V, Sijački A, Gregorić P and Bajec D: Glissonean pedicle approach in major liver resections. Hepatogastroenterology 59: 1896-1901, 2012.

29. Giordano M, Lopez-Ben S, Codina-Barreras A, Pardina B, Falgueras L, Torres-Bahi S, Albiol M, Castro E and Figueras J: Extra-Glissonian approach in liver resection. HPB (Oxford) 12: 94-100, 2010

30. Delis SG, Bakoyiannis A, Karakaxas D, Athanassiou K, Tassopoulos N, Manesis E, Ketikoglou I, Papakostas P and Dervenis C: Hepatic parenchyma resection using stapling devices: Peri-operative and long-term outcome. HPB (Oxford) 11: 38-44, 2009.

31. Schemmer P, Friess H, Hinz U, Mehrabi A, Kraus TW, Z'graggen K, Schmidt J, Uhl W and Büchler MW: Stapler hepatectomy is a safe dissection technique: Analysis of 300 patients. World J Surg 30: 419-430, 2006.

32. Nomi T, Hokuto D, Yoshikawa T, Kamitani N, Matsuo Y and Sho M: Clamp-crush technique for laparoscopic liver resection. Ann Surg Oncol 28: 866, 2021.

33. Kim KH, Kim HS, Lee YJ, Park KM, Hwang S, Ahn CS, Moon DB, Ha TY, Kim YD, Kim KK, et al: Clinical analysis of right anterior segmentectomy for hepatic malignancy. Hepatogastroenterology 53: 836-839, 2006.

34. Dagher I, Gayet B, Tzanis D, Tranchart H, Fuks D, Soubrane O, Han HS, Kim KH, Cherqui D, O'Rourke N, et al: International experience for laparoscopic major liver resection. J Hepatobiliary Pancreat Sci 21: 732-736, 2014.

35. Nitta H, Sasaki A, Fujita T, Itabashi H, Hoshikawa K, Takahara T, Takahashi M, Nishizuka S and Wakabayashi G: Laparoscopy-assisted major liver resections employing a hanging technique: The original procedure. Ann Surg 251: 450-453, 2010.

36. Chen TH, Yang HR, Jeng LB, Hsu SC, Hsu CH, Yeh CC, Yang MD and Chen WT: Laparoscopic liver resection: Experience of 436 cases in one center. J Gastrointest Surg 23: 1949-1956, 2019.

37. Ciria R, Gomez-Luque I, Ocaña S, Cipriani F, Halls M, Briceño J, Okuda Y, Troisi R, Rotellar F, Soubrane O and Abu Hilal M: A systematic review and meta-analysis comparing the short- and long-term outcomes for laparoscopic and open liver resections for hepatocellular carcinoma: Updated results from the European guidelines meeting on laparoscopic liver surgery, Southampton, UK, 2017. Ann Surg Oncol 26: 252-263, 2019. 
38. Ciria R, Cherqui D, Geller DA, Briceno J and Wakabayashi G: Comparative short-term benefits of laparoscopic liver resection: 9000 cases and climbing. Ann Surg 263: 761-777, 2016.

39. Chan AC, Poon RT, Cheung TT, Chok KS, Dai WC, Chan SC and Lo CM: Laparoscopic versus open liver resection for elderly patients with malignant liver tumors: A single-center experience. J Gastroenterol Hepatol 29: 1279-1283, 2014.

40. Nguyen KT, Gamblin TC and Geller DA: World review of laparoscopic liver resection-2,804 patients. Ann Surg 250: 831-841, 2009.

41. Zhao G, Hu M, Liu R, Xu D, Ouyang C, Xu Y, Jiao H, Wang B and $\mathrm{Gu} \mathrm{X}$ : Laparoendoscopic single-site liver resection: A preliminary report of 12 cases. Surg Endosc 25: 3286-3293, 2011.

42. Lefor AT and Flowers JL: Laparoscopic wedge biopsy of the liver. J Am Coll Surg 178: 307-308, 1994.

43. Buell JF, Cherqui D, Geller DA, O'Rourke N, Iannitti D, Dagher I, Koffron AJ, Thomas M, Gayet B, Han HS, et al: The international position on laparoscopic liver surgery: The Louisville Statement, 2008. Ann Surg 250: 825-830, 2009.

44. Scuderi V and Troisi RI: Tissue management with tri-staple technology in major and minor laparoscopic liver resections. Int Surg 99: 606-611, 2014.

45. Nomi T, Fuks D, Govindasamy M, Mal F, Nakajima Y and Gayet B: Risk factors for complications after laparoscopic major hepatectomy. Br J Surg 102: 254-260, 2015.

46. Vibert E, Perniceni T, Levard H, Denet C, Shahri NK and Gayet B: Laparoscopic liver resection. Br J Surg 93: 67-72, 2006

47. Linden BC, Humar A and Sielaff TD: Laparoscopic stapled left lateral segment liver resection-technique and results. J Gastrointest Surg 7: 777-782, 2003.

48. Buell JF, Gayet B, Han HS, Wakabayashi G, Kim KH, Belli G, Cannon R, Saggi B, Keneko H, Koffron A, et al: Evaluation of stapler hepatectomy during a laparoscopic liver resection. HPB (Oxford) 15: 845-850, 2013.

49. Sucandy I, Durrani H, Ross S and Rosemurgy A: Technical approach of robotic total right hepatic lobectomy: How we do it? J Robot Surg 13: 193-199, 2019.
50. Hasegawa Y, Nitta H, Takahara T, Katagiri H, Kanno S and Sasaki A: Pure laparoscopic living donor hepatectomy using the Glissonean pedicle approach (with video). Surg Endosc 33: 2704-2709, 2019

51. Arkadopoulos N, Gemenetzis G, Danias N, Kokoropoulos P, Koukopoulou I, Bartsokas C, Kostopanagiotou G and Smyrniotis V: Cost-effective surgical management of liver disease amidst a financial crisis. World J Surg 40: 1695-1701, 2016.

52. Lin CW, Tsai TJ, Cheng TY, Wei HK, Hung CF, Chen YY and Chen CM: The learning curve of laparoscopic liver resection after the Louisville statement 2008: Will it be more effective and smooth? Surg Endosc 30: 2895-2903, 2016.

53. Zhang EL, Liang BY, Chen XP and Huang ZY: Severity of liver cirrhosis: A key role in the selection of surgical modality for Child-Pugh A hepatocellular carcinoma. World J Surg Oncol 13: 148,2015

54. Tanaka K, Shimada H, Matsumoto C, Matsuo K, Nagano Y, Endo I and Togo S: Anatomic versus limited nonanatomic resection for solitary hepatocellular carcinoma. Surgery 143: 607-615, 2008.

55. Lee KF, Wong J, Cheung YS, Ip P, Wong J and Lai PB: Resection margin in laparoscopic hepatectomy: A comparative study between wedge resection and anatomic left lateral sectionectomy. HPB (Oxford) 12: 649-653, 2010.

56. Santambrogio R, Aldrighetti L, Barabino M, Pulitanò C, Costa M, Montorsi M,Ferla G and Opocher E: Laparoscopic liver resections for hepatocellular carcinoma. Is it a feasible option for patients with liver cirrhosis? Langenbecks Arch Surg 394: 255-264, 2009.

(i) $\odot$ This work is licensed under a Creative Commons Attribution-NonCommercial-NoDerivatives 4.0 International (CC BY-NC-ND 4.0) License. 